\title{
Form of Nerve Impulse and Its Features of Propagation Along the Nerve Fiber Cells Arising from The Bio-Energy in Living Systems
}

\author{
Pang Xiao Feng*, Zeng Hong Juan and Josephine jiang \\ Institute of Life Science and Technology, University of Electronic Science and Technology of China, China
}

Received: 眥 July 13, 2018; Published: 眥July 26, 2018

*Corresponding author: Pang Xiao Feng, Institute of Life Science and Technology, University of Electronic Science and Technology of China, Email: pangxf2006@aliyun.com

\begin{abstract}
We here researched the features of transport of nerve impulse along the nerve fiber using modern theory of molecular biology, in which we first elucidate the properties of structure of the nerve cell and the features of distribution of the charges for the sodium ions and potassium ions in the inner and exterior of the never cell membranes. In practice, their distributions are not uniformity, where the distribution of sodium ions and potassium ions between the inner and exterior of cell-membranes are not same, they are just inverse. Just so, an action electro-potential occurs in the cell-membranes. However, the action electro-potential is only a static impulse, it cannot propagate along the nerve organizations. If the nerve organizations are acted by the bio-energy, which could lead to the periodic variation of these sodium ions and potassium ions in the inner and exterior of the never cell membranes can be varied periodically under the action of bio-energy by the works of sodium pump and potassium pump on the surface of cell membrane, then the nerve impulse can propagate along the nerve fiber cell membranes. Our investigations verify that the bioenergy released from the hydrolyses reaction of adenosine phosphate (ATP) molecules in the cells can play the role. This chemical reaction can release the bio-energy of $0.42 \mathrm{eV}$, which can be transported along the protein molecules to excite the work of se sodium pump and potassium pump. Thus, the propagation of the nerve impulse can be carried out automatically in living systems. This is just the mechanism of propagation of the nerve impulse along the nerve l fiber cel membrane. In this paper we elucidate and research in detain and deeply the mechanism of form of the nerve impulse and its features of propagation.
\end{abstract}

Keywords: Nerve impulse; Nerve membrane; Nerve system; Bio-energy; Protein molecule; ATP molecule; Energy transport

\section{Introduction}

It is known that the nerve system is very important organization in animal and humanity. The animal and humanity cannot live and move without the nerve system. A basical and important function of the nerve system is to transport the nerve information to all organizations for their works by virture of the propagation of nerve impulse. This means that the features of nerve impulse and its $t$ propagation play very important rules in life activities. However, a lot of problems for the nerve imulse, for example, its mechanism of form and generation, its features of propagation and its effects and functions, and so on, have not been researched systematically and deeply up to now. But, these problems are very important in nerve sciences and biology because we cannot understand and know clearly the theorem of work of each organization and their mechanism of cooperation and synergistic effects. Therefore, to research them are quite necessary. In this paper we describe and elucidate first molecular structure of nerve cell.

\section{The characterizations of structure of nerve cells}

The form of the action electric-potential in nerve membranes in the nerve cell-membranes are first described, it is a nerve impulse and can be formed by periodic and ruled changes of nonuniform distribution of the sodium and potassium ions in the inner and surface of nerve cell-membranes. The nerve impulse can be also transported along the nerve fiber membranes under action of periodic works of sodium pump and potassium pump arising from the bio-energy released from the hydrolyses reaction of ATP molecules, which is transported by Pang's soliton along the protein molecules. The experiments verified that there is not the nerve impulse, or the action electric- potential without the works of sodium pump and potassium pump, or the bioenergy. We investigated further the properties of transport

of the nerve impulse along the nerve Ferber membranes. 
The unit of the nervous system in animals is the nerve cell or the neuron. It is the unit of structure and function of nervous system, its main function is to accept process and transfer the nerve information, to complete the functions of the nervous system. It consists of nerve cells having different sizes and forms, which is shown in Figure 1. In addition to general structure of cell, which contains the membrane, cytoplasm and nuclear, the neuron or nerve cell is also composed of the membrane, cytoplasm and nuclear, but their sizes and shapes are different those of general cell. In particle, the neuron is a fiber cell and contains the cell-body and many bumps, such as one axon and many dendrite, which are issued from the cell-body, in the cytoplasm there are an austenite (or Nissl substances), which is an active part and the position of syntheses of protein molecules. At the same time, the neurons involve also the large number of microtube and micro-trabecular, which is formed through accumulated by a plenty of large number of polarized protein molecules, which resembles with a polymer formed by many dipoles in accordance with the style of order arrangement, it is also an acted site of the narcotics of central nervous. In fact, the size and shape of different neurons are very distinct, but their structures are almost same, they can all be represented by (Figure1) [1,2]. The branch of the axon is called the lateral branch.

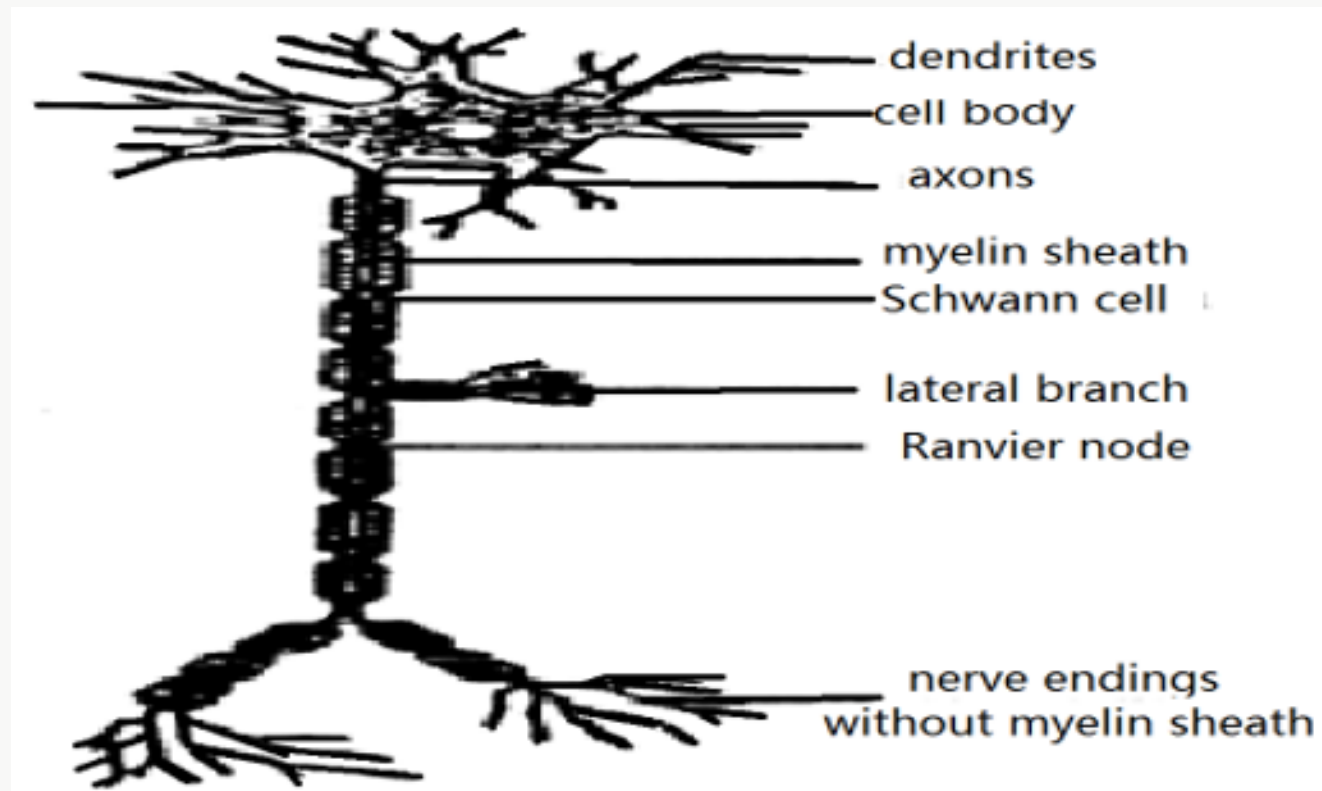

Figure 1: The structure of the neuron.

The lengths of axon and lateral branch are not same, their differences are also very large, some are very short, which are about several micrometers, but the lengths of most of

bumps in the neurons are very great, such as their lengths may reach $1 \mathrm{~m}$ in the persons and animals. When the axons are contacted with other cell bodies, some structures of myelin sheathe may occur in the neurons for the persons and animals. However, the neurons without myelin sheathes exist also in some organisms. Experiments verified that thirty-six carbon olefine acid (DHA) promotes the forms of the myelin sheathes in the processes of extension and metabolism of the neural axons. The axon endings in Figure1 can both output electric-information and release the neurotransmitter to affect other neurons or non-nerve cells. This means that the axons and lateral branches can all link with other -nerve cells by the synapseses and another cell body. Thus, a completely neural signal transmission system is formed in this case. The dendrites and axons most vertebrates' neurons and membranous organelles, which can synthesis cell bodies and extra telecom and neurotransmitter release, in order to affect the close contact with it another, the axons and all collateral can be a nerve cell through the synapse and another cell precursor and form a neural signal transmission system. The dendrites and axons of most neurons in vertebrates are issued from their cell bodies coated in the center of the cells. The cell-body contains the nuclear and organelles having feature of membranous to responsible for synthesizing and processing protein molecules. At the same time, the synapses of the neuron are often located between the dendrites and cell-body, this means that the cell-body participate also the information integration and collection. The experimental results showed that the dendritic structure of a special nerve cells can enhance the calculation functions of nerve cells in the [1].

\section{The basic structure of nerve systems}

The unit of the nervous system in animals is the nerve cell or the neuron. It is the unit of structure and function of nervous system, its main function is to accept, process and transfer the nerve information, to complete the functions of the nervous system. It consists of nerve cells having different sizes and forms, which is 
shown in Figure 1. In addition to general structure of cell, which contains the membrane, cytoplasm and nuclear, the neuron or nerve cell is also composed of the membrane, cytoplasm and nuclear, but their sizes and shapes are different those of general cell. In particle, the neuron is a fiber cell and contains the cell-body and many bumps, such as one axon and many dendrite, which are issued from the cell-body, in the cytoplasm there are an austenite (or Nissl substances), which is an active part and the position of syntheses of protein molecules. At the same time, the neurons involve also the large number of micro-tube and micro-trabecular, which is formed through accumulated by a plenty of large number of polarized protein molecules, which resembles with a polymer formed by many dipoles in accordance with the style of order arrangement, it is also an acted site of the narcotics of central nervous. In fact, the size and shape of different neurons are very distinct, but their structures are almost same, they can all be represented by Figure 1 [1-2].

The branch of the axon is called the lateral branch. The lengths of axon and lateral branch are not same, their differences are also very large, some are very short, which are about several micrometers, but the lengths of most of bumps in the neurons are very great, such as their lengths may reach $1 \mathrm{~m}$ in the persons and animals. When the axons are contacted with other cell bodies, some structures of myelin sheathe may occur in the neurons for the persons and animals. However, the neurons without myelin sheathes exist also in some organisms. Experiments verified that thirty-six carbon olefine acid (DHA) promotes the forms of the myelin sheathes in the processes of extension and metabolism of the neural axons. The axon endings in Figure 1 can both output electric-information and release the neurotransmitter to affect other neurons or non-nerve cells. This means that the axons and lateral brarches can all link with other -nerve cells by the synapseses and another cell body. Thus, a completely neural signal transmission system is formed in this case.

The dendrites and axons most vertebrate's neurons and membranous organelles, which can synthesis cell-bodies and extra telecom and neurotransmitter release, in order to affect the close contact with it another, the axons and all collateral can be a nerve cell through the synapse and another cell precursors and form a neural signal transmission system. The dendrites and axons of most neurons in vertebrates are issued from their cell bodies ocated in the center of the cells. The cell-body contains the nuclear and organelles having feature of membranous to responsible for synthesizing and processing protein molecules. At the same time, the synapses of the neuron are often located between the dendrites and cell-body, this means that the cell-body participate also the information integration and collection. The experimental results showed that the dendritic structure of a special nerve cells can enhance the calculation functions of nerve cells in the [1].

\section{The form of the synaptic structures and its properties}

The synaptic structures have very important roles in the nerve activities, its structure and features are worth to study seriously. Its form is described as follows. The axons at the grown position in the neuron are grown and extended some larger cones, in this case their cone end of the protuberance or bumps can contact or combine to form a function wide organ with the bottom of axons or dendrites or cell-body of neighboring neuron. The organ between two neurons is referred to as the synaptic as shown in Figure1 [1-6]. Synaptic is a contact area and small gap having exceptional function, which can transport and transfer the neural signals from one neuron to another neuron. However, they are not continuous in the protoplasm. The synaptic is the association origin of function between two nerve cells and the position of contact of structures. Through the associations and their works, the neurons and receptor cells as well as the effect or cells are associated together to form a complete system of transmission of neural signals. There are not the transmission and transport of neural signals without the synaptic. Therefore, the synaptic is a key origin in neurobiology.

It is estimated that the human brain has about $10^{14} \sim 10^{15}$ synapses. Single synaptic is a complex of incoming - outgoing unit of signs, which is just a basic feature of the neurons. All neurons are all linked together through them. The related linkage between two neurons or seeking, the synaptic contains three parts: the before-part of synaptic of active cells or their protuberance given out, after-part of synaptic of cells accepted activation or their protuberance and the part or gap between them. Therefore, the correct forms of linkage of the synapses require not only the right guidance of growth of the protuberances along the surface of cells and extracellular pathways, but also the specific identification between the presynaptic and postsynaptic cells. At the same time, it demands also stability of synapses in the activation in the motion of time and space. In biology, the synaptic connections, or speaking shape or form of e synaptic are various. If an axon or a lateral branch are terminated in another neurons, then it is called the axis body synapse, but the synapnic between the axons and dendrites is called the axon-dendrite synapntic, the synaptic between two axons is the axon-axon synaptic. If one axon is terminated on the skeletal muscle fibers, then it is referred to as the neuromuscular endplate. A basic feature of the synaptic transmission are the valve and one single direction of the transfer, its information of transfer can be integrated and has a plasticity, i.e., its efficiency and functions are variable, the capacity of transferred information are higher and have the multi-purposes. These features are the foundation of orderly neural activity and learning and memory of animals and persons [1-2].

In the central nervous system, the typical presynaptic and postsynaptic compositions are closely associated on the membrane 
each other, which will lead to the alienation of contacted membrane, thus the two-layer membranes should be separated by a narrow synaptic gap. The synaptic gap is an analogue matter of basement membrane, which is derived from the before- and after- membranes. The materials in the synaptic gap are all the protein molecules, which involved the alkaline amino acid with high concentrations, its interstitial matter has the sialic acid and glycoprotein, etc. In the side of pre-synaptic there are the synaptic corpuscles of swollen and tapered protuberance, in which many vesicles with a lot of neurotransmitter of high concentration are contained.

The synaptic has three types of chemical synaptic and electric synaptic and their hybrid. The chemical synaptic and electric synaptic, specially, the former exist widely in all animals and Human beings, but the latter exists only in the invertebrate and lower vertebrate animals, their nerve in formations are transferred by means of the variations of electric potentials among the gap junctions of synapses, in which the circuit impedance between two neurons is very larger, but their electric-potential are lower. The chemical synaptic is widely existent in the nervous systems in the breastfeeding animals, its transport of the nerve in formations are carried out by the release and transport of chemical materials, which are called as the neurotransmitters, or messenger, such as the endocrine hormone, neural hormone of cells, etc. Chemical synapses are the most complicated synapses in the nervous system, their connections possess a specificity, such as its transport of nerve information has an exact direction from one neurons to another neurons and polarity. Its polarity is mainly determined by a group of synaptic vesicles and paralleling membranes. There is a gap with the size of $20 \mathrm{~nm}$ between the membranes of two neurons in the parallel membrane, its density of material is larger, especially for the pair-side membrane involving the protuberance of synaptic vesicles.

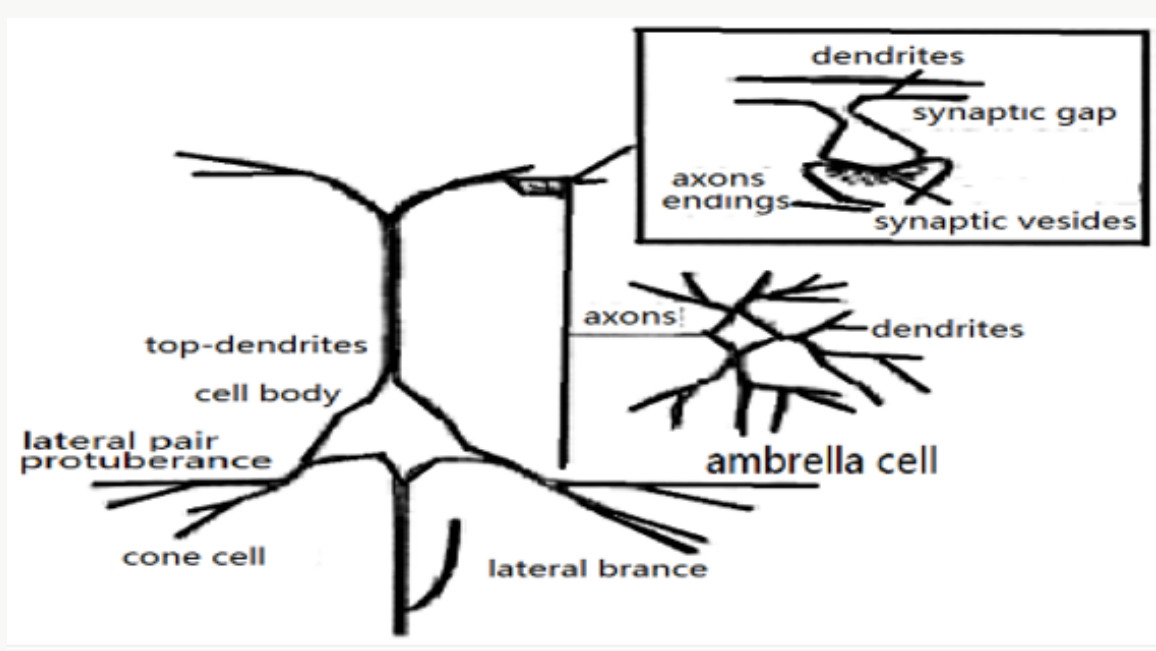

Figure 2: synaptic structure in the neuron [1-2].

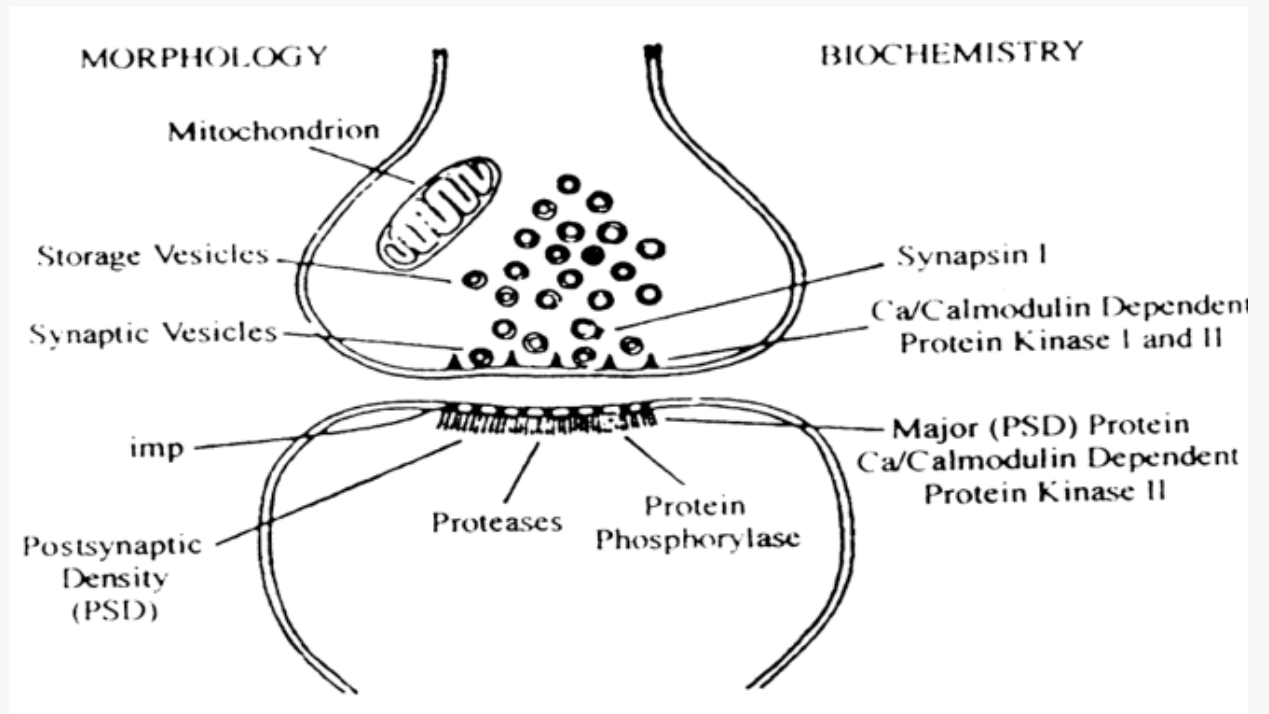

Figure 3: The configuration and biochemical properties of chemical synapses, where mp denotes the corpuscles in the membrane, PSD denotes the thickening of postsynapses [1,2,7]. 


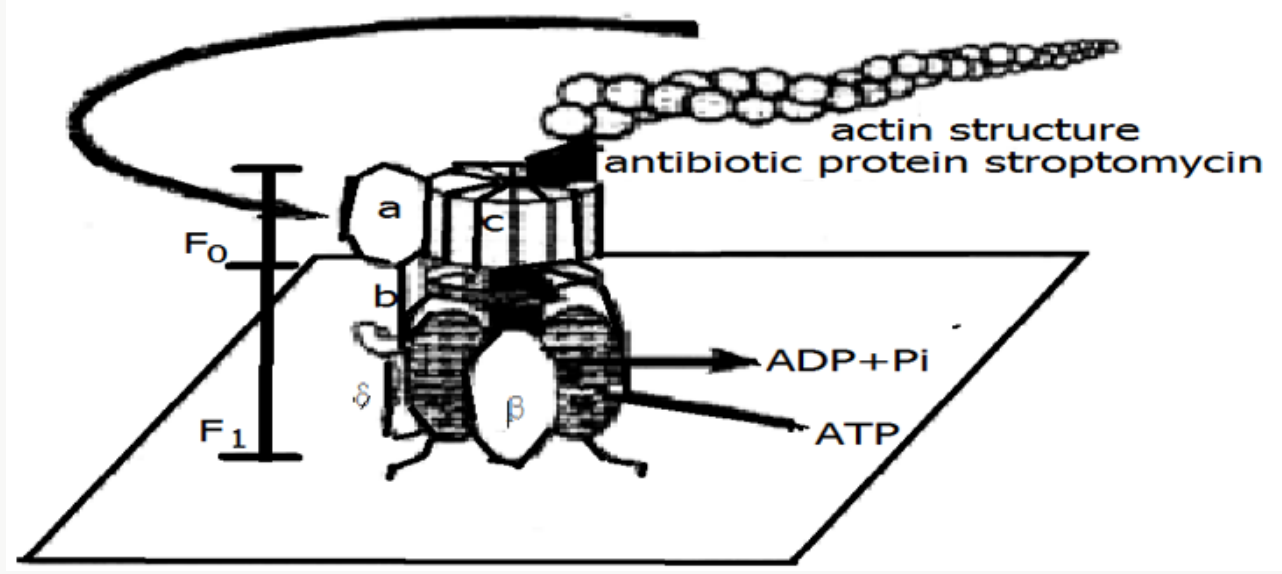

Figure 4: The structure of rotary motor-synthase [1-4].

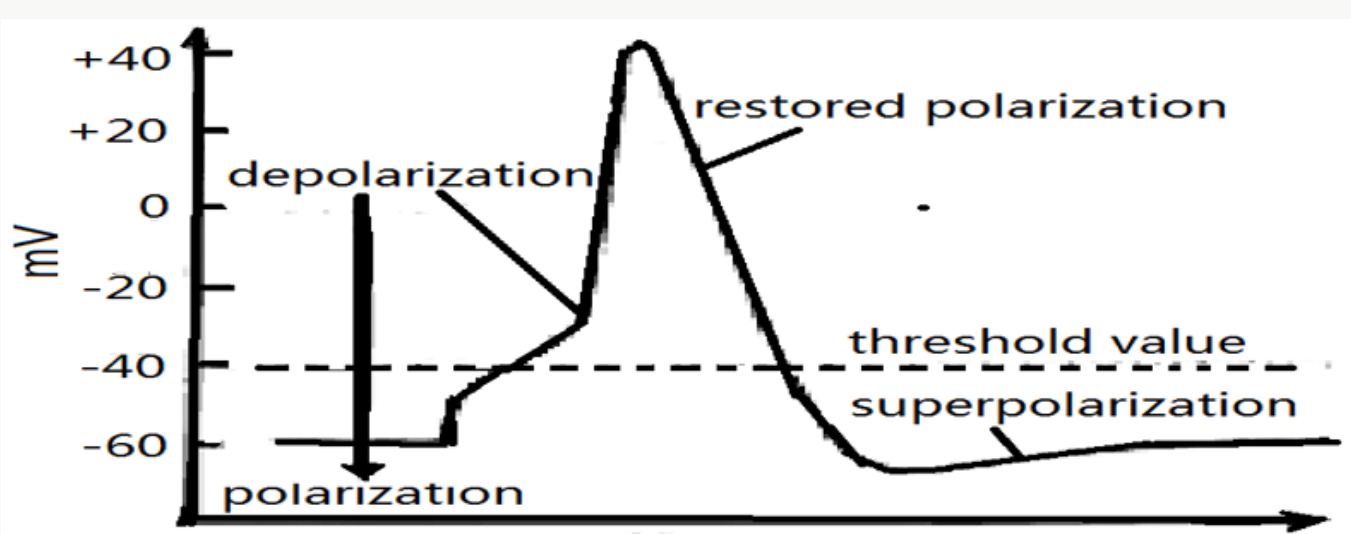

time

Figure 5: The action electric-potential of cell membrane 1 [1-3,7-10].

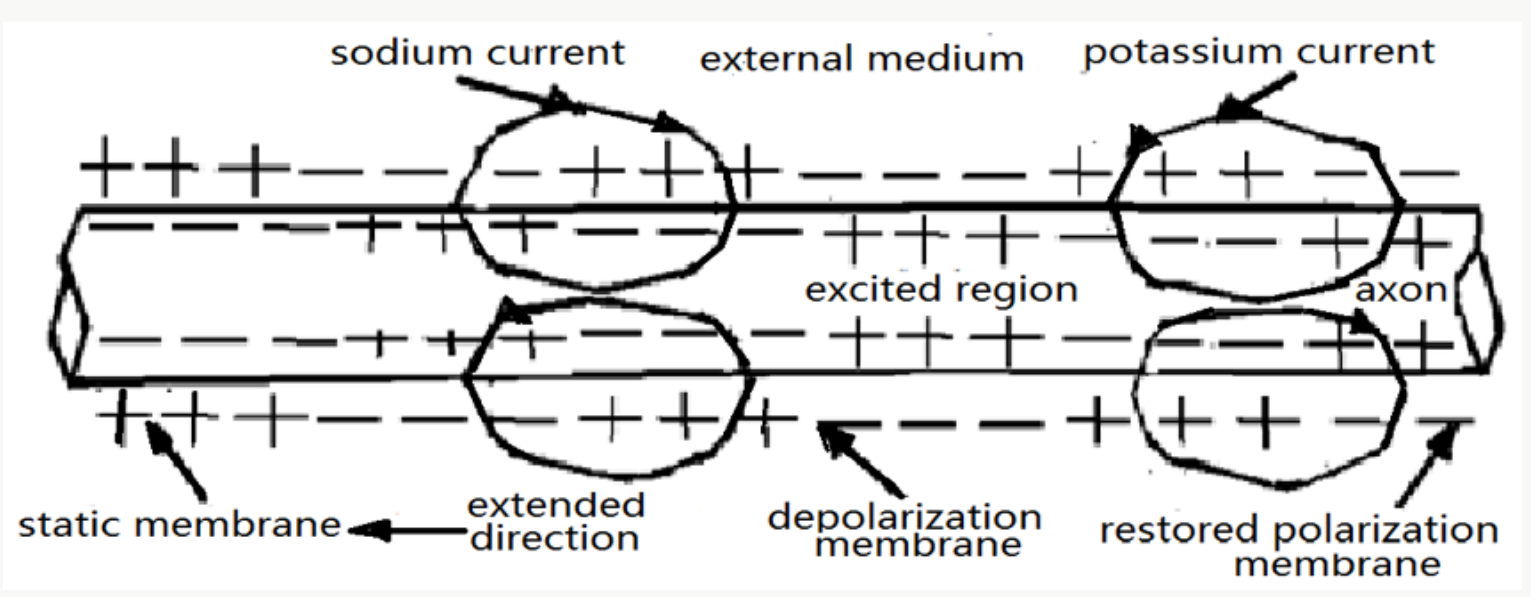

Figure 6: Form of nerve excitement in the nerve system [1-2,10].

The comparison of properties of chemic and electric synapses is shown in Table 1. The structure of the chemical synapses is shown in Figure 3. It contains the submicro-structures of the synaptic endplate, synaptic vesicles, front membrane of synaptic, after-membrane of synaptic, synaptic vesicles and postsynaptic membrane. The synapsin 1 in the front membrane is a protein molecule and a heterodimer constructed by the protein molecules with the molecular weight of $166 \mathrm{kDa}$ and are related to the synaptic vesicles. After the synapsin 1 is in phosphorylation state, it is separated from the vesicles membranes and combination with the cytoplasmic membrane through cell skeleton, thus some neurotransmitters are released in this case. The phosphorylation 
is agan catalyzed by the calcium/calcium modulin dependent protein kinase 1 , which can have activated by the internal flow $\mathrm{Ca}^{2+}$, when a nerve impulse occurs. The determined compositions in the postsynaptic membrane are the membrane receptor, thickening of postsynaptic (PSD) and the enzymes, which can make PSD phosphorylation and dephosphorylation. The thickening of postsynaptic is mostly formed by the cytoskeleton of the membrane, which contains the many microtubules, nerve filaments, and combines many proteins and proteolytic enzymes. The main composition of PSD is the peptides of $50 \mathrm{kDa}$ or proteins. The above components are all shown in Figures $2 \& 6$ in which the calcium/calmodulin protein kinase II (CaM II) is a major biochemical component of synapses and exists mainly in the presynaptic endings and PSD, and it is also one of the homologous kinase family. It is consisted of 12 subsites with weighs of $50 \sim 60$ $\mathrm{kDa}$, which is a larger polymer of proteins, its basical function is itself phosphorylation of subunits. So-called itself phosphorylation is just make the kinases have a function of switch, then the kinas can still maintain itself bio-activity after short calcium-information signals are accepted. This feature is very advantageous to the longtime activity of the postsynaptic and their variations. Otherwise, there are another some scaffolding proteins in the postsynaptic, which contains the actins and the protein of $43 \mathrm{kDa}[1-2,7]$.

Table 1: The comparisons of properties of chemic and electric synapses [1-2,7].

\begin{tabular}{|c|c|}
\hline The chemic synapses & The electric synapses \\
\hline $\begin{array}{c}\text { The affection of action potential of the } \\
\text { presynaptic on electric potential of after } \\
\text { membranes is small }\end{array}$ & $\begin{array}{c}\text { The action potential of } \\
\text { presynaptic is a direct } \\
\text { factor of the transport }\end{array}$ \\
\hline $\begin{array}{c}\text { Presynaptic potential caused } \\
\text { many synchronous releasing of } \\
\text { neurotransmitters by the small bubble } \\
\text { under action of cooperation of ca2 }\end{array}$ & Without this effect \\
\hline $\begin{array}{c}\text { The releases of euro transmitter produce } \\
\text { the appearing of local and different } \\
\text { potentials of postsynaptic membrane, its } \\
\text { sizes can be integrated, the postsynaptic } \\
\text { potential can be inverted when it is varied }\end{array}$ & $\begin{array}{c}\text { The postsynaptic potential } \\
\text { has noen inverted, when } \\
\text { it is varied }\end{array}$ \\
\hline $\begin{array}{c}\text { There are synaptic vesicles in this case } \\
\text { It can be both excited and inhibited }\end{array}$ & Without synaptic vesicle \\
\hline $\begin{array}{c}\text { It is easily influenced in chemical factors } \\
\text { space and time, it has also an integrated } \\
\text { effect }\end{array}$ & It is not influenced \\
\hline $\begin{array}{c}\text { Its effect can be sux }(0.1) \text { mmarized in the } \\
\text { Integration }\end{array}$ \\
\hline
\end{tabular}

The nerve excitation and action electric-potential as well as use of bio-energy in the nerve systems

\section{The resting electric-potential of cell membrane and its mechanism}

What is nerve excitement? It is that the nervous tissues give out a strong reaction, when its strength reaches or exceeds the certain threshold. This phenomenon appears also in the muscle tissue. Its main feature and performance are that the electric-potential across the cytoplasmic membranes generated a fast variation. In this case the strength of variation of electric-potential is used to characterize and mark the state and intensity of the nerve-excitation. We here will discuss the excitation states of the nerve and its features and representations in the nerve system.

As it is known, the molecular structure of the neurons is also same with those of general cells, although their configurations have some differences, such as the distributions of the ions of $\mathrm{Na}^{+}$, $\mathrm{K}^{+}$and $\mathrm{Cl}^{-}$on the membrane of the neurons are same with those of other cells, i.e., they are all non-uniformly distributed in the side and outside of the cell membrane, in which the number of $\mathrm{Na}^{+}$ions in its outside are more than those in its inside, but the distributions of $\mathrm{K}^{+}$and $\mathrm{Cl}^{-}$are just inverse, namely he numbers of $\mathrm{K}^{+}$and $\mathrm{Cl}^{-}$ions in the inside of cell membrane are more than those in its outside. This means that an electric potential is formed and appeared on the cell membrane due to the non-uniform of distribution of the charges or ions in the inside and outside of cell membrane, this is necessary to results in the differences of permeability of ions to the cell membrane. Thus the electric-potential is formed, which is called the resting potential of cell or the potential of numbers, its size is about $-40--70 \mathrm{mV}$, i.e., the electric-potential in inside of cell membrane is lower than that in its outside, which was verified by the experiments and theory of biophysical theory. These results manifested clearly there are many ions in inside and outside of cell membrane, but the numbers for different ions are different. These phenomena indicated that there are also some channels of the ions on the cell membranes, which are formed by membrane -spanning proteins molecules, but their electric resistances of different ions are different. The above resting potential of cell membrane can be determined and obtained using the above results.

In particle, in the nervous tissues the compositions of the inside and outside electrolytes are also different, such as the concentrations of $\mathrm{Na}^{+}$and $\mathrm{Cl}^{-}$are more many in the outside of the axons of active- squid, but much less in its, but the distribution of $\mathrm{K}^{+}$ is just opposite. In the resting state, the charge distributions in the inside and outside of the cell membrane in the nerve fibers is still negative in the interior and position in the exterior, thus its electric potential is about $70 \mathrm{mV}$, its membrane resistance is $10^{3} \Omega / \mathrm{cm}^{3}$, its membrane capacitance is $1 \mathrm{mf} / \mathrm{cm}[1-3,7]$.

The huge differences of permeabilities and resistances of these ions on the cell membrane in the squid animal lead on to their large distinctions of electric-potentials. The researches indicated the differences of the potentials can be represented by $\mathrm{P}_{\mathrm{K}}: \mathrm{P}_{\mathrm{Na}}: \mathrm{P}_{\mathrm{cl}}$ $=1: 0.04: 0.45$, where $\mathrm{P}_{\mathrm{K}} \mathrm{P}_{\mathrm{Na}}$ and $\mathrm{P}_{\mathrm{Cl}}$ are the electric-potentials of $\mathrm{K}^{+}, \mathrm{Na}^{+}$and $\mathrm{Cl}^{-}$ion on the membrane. This result exhibited that $\mathrm{K}^{+}$ plays important part in the form of resting electric potential of huge axons in the squid. In this case, most of the $\mathrm{K}^{+}$ions are in free states, but half of $\mathrm{Na}^{+}$ions either are in the combined states or form some products in the cells, otherwise, $\mathrm{Ca}^{2+}$ ions are almost completely 
in the combined states with some negative ions or in organelles (mitochondria, etc.) in the cells. Therefore, the permeability of $\mathrm{Na}^{+}$ on the membrane is small in resting states of cells.

The above properties of permeability of ions can be checked by some experiments, for example, the variations of permeability of ions on the membrane was measured, when some specific inhibitors are added in it. For instance, the water-soluble paralytic poison - tetrodotoxin (TTX), which are extracted from some fishes and newts in California, are added in the gaps between their axons, the experiments found that the channels and corresponding movements of $\mathrm{Na}^{+}$ions in the nerve and muscle fibers are stopped completely. If the TTXs are injected in the axons, the permeability of $\mathrm{Na}^{+}$ions are not changed. However, if the frog toxin (BTX), which is a kind of steroid alkaloids, is injected in the above animals, it will result in the depression of resting electric-potential and increases of the permeability of $\mathrm{Na}^{+}$ions. If the inhibitors of four procedures (TEA) are injected in the axon, then the permeability of $\mathrm{Na}^{+}$ions have been not varied, but it can stop completely the movement of $\mathrm{K}^{+}$across the membrane. These experimental results not only conform the validity of the above results but also exhibited that the movements of $\mathrm{K}^{+}$across the membrane are completely independent relative to that of $\mathrm{Na}^{+}$ions. This represented also that the penetration abilities of different ions on the membranes have a strong specificity. The identified effects of the inhibitors of TTX and TEA on the permeability of $\mathrm{Na}^{+}$and $\mathrm{K}^{+}$make that we can assume that the transport of the ions across the membranes are carried out by protein - lipid compounds or ion channels. Thus each ion has itself special channel of ions. The tetrodotoxins stopped the transport of $\mathrm{Na}+$ because they have closed the channels of sodium ions in one square micron. The experimental measures indicated that there are 100 channels of $\mathrm{Na}^{+}$in one square microns. In the resting or inactivity state, it is estimated experimentally that the conductivity of $\mathrm{Na}^{+}$in a sodium channel is about $4^{\times} 10^{-12} \Omega^{-1}$, but about $12^{\times} 10^{-12} \Omega^{-1}$ for $\mathrm{K}^{+}$in the potassium channe [1-2,7].

On the other hand, in resting state the permeability of potassium ions across the excited membrane of cell is larger than that of sodium ions. In addition, some macromolecules or ions with negative charges inside cell membrane cannot penetrate the membrane, based on these results we can establish the relationship of these ions between the inside and outside cell membranes. In this case, we mark these different ions in inside and outside cell membrane by the subscript 1,2 . Because again there are also some ions with negative charge of nonvalenced as mentioned above, which is denoted by $\mathrm{P}^{-}$, Thus we can represented the relationship of the chemical potentials of these ions in outside and inside cell membrane in the equilibrium state on the basis of the conditions of equality of the chemical potentials and charges in the inside and outside the cell membrane of the ions of $\mathrm{K}^{+}$and $\mathrm{Cl}^{-}$, which is denoted[1] by

$$
\mu_{k 1}^{+}=\mu_{k_{2}}^{+} \mu_{d 1}^{-}=\mu_{k_{2}}
$$

where $\mu_{i}$ is the chemical potential of the ion. In the ideal solution, the chemical potentials can be represented by

$$
\mu_{i}=\mu_{i}^{(0)}+R T \ln C_{i}+Z_{i} F \phi
$$

where $\mu_{i}^{(0)}$ is the chemical potential in the standard state, $\mathrm{C}_{\mathrm{i}}$ is the concentration of the ion in the solution, $\mathrm{Z}_{\mathrm{i}}$ is chemical valence of the ion, $\phi$ is the resting electric -potential of corresponding element, F is Faraday constant.

From Eq. (1) we can obtain

$$
\begin{aligned}
& \mu_{K_{1}^{+}}^{(0)}+R T \ln \left[K^{+}\right]_{1}+F \phi_{1}=\mu_{K_{2+}}^{(0)}+R T \ln \left[K^{+}\right]_{2}+F \phi_{2} \\
& \mu_{C l_{1}^{-}}^{(0)}+R T \ln \left[C l^{-}\right]_{1}-F \phi_{1}=\mu_{C l_{2}}^{(0)}+R T \ln \left[C l^{-}\right]_{2}-F \phi_{2}
\end{aligned}
$$

The following results can be obtained from Eqs. (3)-(4)

$$
\frac{\left[\mathrm{K}^{+}\right]_{1}}{\left[\mathrm{~K}^{+}\right]_{2}}=\frac{\left[\mathrm{Cl}^{-}\right]_{2}}{\left[\mathrm{Cl}^{-}\right]_{1}}=v
$$

Equation (5) is called as Gibbs-Dsnnan condition is Donnan proportional constant.

From Eqs. (3)-(4) we can gain the potential of the ion across the membrane

$$
\phi=\phi_{1}-\phi_{2}=-\frac{R T}{F} \ln \frac{\left[K^{+}\right]_{1}}{\left[K^{+}\right]_{2}}=\frac{R T}{F} \ln \frac{\left[C l^{-}\right]_{1}}{\left[C l^{-}\right]_{2}}=\frac{R T}{F} \ln v
$$

Because the resting potential is mainly caused by the permeability of $\mathrm{K}^{+}$ions, if the concentration $\mathrm{y}$ of potassium ions $\left[\mathrm{K}^{+}\right]_{2}=20 \mathrm{mmol}$ and $\left[\mathrm{K}^{+}\right]_{1}=100 \mathrm{mmol}$ are inserted in Eq. (5)-(6) we can easily obtain $\phi_{\mathrm{k}}=-75 \mathrm{mV}$, this is basically consistent with the experimental value of resting electric potential value of $-70 \mathrm{mV}$ measured. This verified clearly that the distribution of the ions on the cell membrane described mentioned above are correct. This conformed strongly that the resting electric-potential of the membrane is produced by the permeability of $\mathrm{K}^{+}$ions [1,2,7-9].

If second condition :

$\left[K^{+}\right]_{1}=\left[C^{-}\right]_{1}+\left[P^{-}\right]$and $\left[K^{+}\right]_{2}=\left[C^{-}\right]_{2}=C_{2}$

is used we can find out the following relationship

$$
v=\frac{\left[P^{-}\right]}{2 C_{2}}+\sqrt{\left(\frac{\left[P^{-}\right]}{2 C_{2}}\right)^{2}+1} .
$$

\section{The forms and works of sodium pump and potassium pump in the nerve membrane and use of bio-energy in the nerve systems}

As it is known from the above results, the ions of $\mathrm{Na}+, \mathrm{K}^{+}$and $\mathrm{Cl}^{-}$can be displaced and flowed along the ionic channels on the cell membranes due to their nonuniformities of distribution, 
which promote the fluxes of $\mathrm{Na}^{+} \mathrm{K}^{+}$and $\mathrm{Cl}^{-}$along their directions of decrease of electric chemical potentials. Thus some ion-currents are occurred on the membrane. This results in just occurring of the electric signs in the cells. Obviously, the electric signs are generated due to the open and close of the ionic channels. In the normal state $\mathrm{Na}^{+}$ions are shifted from the outside cell membrane to its interior, but $\mathrm{t}$ the displacement of $\mathrm{K}^{+}$and $\mathrm{Cl}^{-}$are just inverse and to the outside membrane from its interior. Very clearly, these fluids will induce the variations of distribution of these ions from the non-uniform state to the uniform state, thus the electricsigns of the cells will decreased, which are not advantageous for the growth of the cell or life. In this case it is quite necessary to maintain the ion concentration gradient on both sides of the cell membrane mentioned above. In order to gain this purpose, its basic method to construct and grow some ion pumps, such as the sodium pump and potassium pump, which can hand and carry $\mathrm{Na}^{+}$ions to the outside membrane from inside membrane, and can hand and carry also $\mathrm{K}^{+}$ions to the inside membrane from outside membrane, respectively. Thus the nonuniform distribution of $\mathrm{Na}^{+}$ions and $\mathrm{K}^{+}$ ions or their gradients of concentration on the cell membrane can be always maintained or restored also in active life bodies using these ionic pumps. In other words these ion pumps can provide the bio-energies to carry away these ions to specifying positions to maintain the gradients of concentration of these ions in both sides of the cell membrane to a certain level, then all life activities can remain and continue, if the bio-energy is supplied enough. Or else, the life activities will be completely stopped. Therefore we can say that the $\mathrm{Na}^{+}$and $\mathrm{K}^{+}$pumps and the supply of bio-energy play very important roles in the life activities [1-2,7-9].

However, how can these ion pumps be constituted and grown? Very clearly, the work can be carried out and finished by some special protein molecules or enzymes, which can also bring away and carry away these ions to specifying positions. These proteins or enzymes are some rotary motor -proteins or ATP synthases, such as the rotary motor -protein as shown in Figure 4, DNA helicase motor and RNA polysaccharide enzymes motor. We here will elucidate the structures and functions of the ion pump in the ATP s synthase in Figure 4, which can shoulder the work of syntheses of ATP and can provide the bio-energy. The enzyme is composed of the part of proton conduction, $\mathrm{F}_{0}$, and it's part of promotion, $\mathrm{F}_{1}$. When the protons traverse the F0, ATP molecules will be synthesized in $\mathrm{F}_{1}$, thus the bio-energies generate immediately by means of the hydrolyses reaction of ATP molecules. This reaction is reversible. In this case, the bio-energy can promote the protons to move along the inverse directions, if the hydrolyses reaction of ATP molecules occurs in $\mathrm{F}_{1}$. Because $\mathrm{F}_{1}$ can catalyze the hydrolysis of ATP molecules, then it is called as $\mathrm{F}_{1}$-ATP enzyme. The ATP synthesized enzymes exist also in the inner membrane of the mitochondrial and the serosa in the bacteria as well as capsule membrane in the chloroplasts.
In the process of syntheses of ATP molecules, the chemical potential of the protons must be consumed and expended, but the chemical potential can be complemented by the biological membrane including the light-chemical systems. When ATP enzymes rotate the protons along the inverse direction, the hydrolyses of ATP molecules occur, thus the electric-chemical potential of the protons is also formed in this case. However, the transmission mechanism of the protons in the reverse reaction is always related to the hydrolysis of ATP molecules. As it is known, the syntheses and hydrolyses of ATP molecules are carried out by the $\mathrm{F}_{1}$-ATP enzymes on the enzyme membrane. $\mathrm{F}_{1}$-ATP enzyme has a strong activity of hydrolyses of ATP molecules, but $F_{0}$ part of the enzyme can also cause the shift of the protons and promote further their movements of across the membranes. The distance between the part of $\mathrm{F}_{1}$-ATP enzyme in the hydrolytic enzymes and the proton transmission area is about $8 \mathrm{~nm}$, they are linked by $\gamma$ sub-base. Therefore, the syntheses and hydrolyses of ATP molecules and proton transmission are completed through the mutual combination among them using the $\gamma$ sub-base, in which the increment of rotation is about $120^{\circ}$, but the direction of $\gamma$ base will be rotated about $120^{\circ}$, when one hydrolysis reaction of ATP molecules is appeared, thus its efficiency is close to $100 \%$.

Obviously, the structure features of rotary motor-ATP synthase and its functions can use to explain and elucidate the mechanism of sodium pump and potassium pump in nerve systems mentioned above, in which the sodium pump carry and bring the $\mathrm{Na}^{+}$from the inside cell membrane to outside cell membrane, but the potassium pump carry and bring the $\mathrm{K}^{+}$from the outside cell membrane to inside cell membrane to maintain the gradients of concentration of these ions in both sides of the cell membrane. These functions are carried out in virtue of its special identification abilities to the sodium and potassium ions through their rotation features and special atomic weighs and moved stats as well as the sizes of combination positions such as $\mathrm{F}_{0}$ and $\mathrm{F}_{1}$ of the motor ATP synthase.

Certainly, the motions and states of the motor ATP synthase are very complicated, if they are in detail investigated. These problems are worth to study deeply and completely. At present, we again elucidated in detail the essences and properties of work of sodium pump and potassium pump in the nerve systems. In practice, the $\mathrm{Na}+$ ions and $\mathrm{K}+$ ions with water flow together along their channels, if these ions move along their ionic channels across the cell membranes. Once they are flowed over the cell membranes, then water will be taken off from these ions. In this case these ions in the dehydrations can be absorbed by other polar groups in these ionic channels. To maintain the ion concentration difference between the inside and outside membrane of the nerve fiber cells at the required level, the sodium pump must work constantly and make the hydrolysis of ATP molecules release continuously the bioenergy in the entire life period. In the nerves systems of the crab, 
about $50 \%$ of metabolic energy were used in the sodium pump in the nerve fibers in the resting state. In the crab the hydrolysis of ATP molecules is together finished by ATP-enzyme, $\mathrm{K}^{+}, \mathrm{Na}^{+}$and $\mathrm{mg}$ ${ }^{2+}$, where ATP-enzyme is a part of the membrane, its other part is in outside cell membrane. In this case there are an interaction between the enzymes in the outside membrane and $\mathrm{K}+$ in the outside cells .

When $\mathrm{K}^{+}$exists, the work of potassium pump will be stopped, the sodium channels are opened duo to the excitation of $\mathrm{Na}^{+}$in this case, then the sodium pump start its work to make or to forces $\mathrm{Na}^{+}$ ions up- shift along the direction of increase of its concentration. In this time, $\mathrm{Na}^{+}$ions are displaced and transferred into the inside membrane by its ionic motions in channels and their interactions with some moved carriers, which guarantees the the gradient of its concentration between both sides of cell membrane and the transport of $\mathrm{Na}^{+}$ions in plasma. On the other hand, $\mathrm{K}^{+}$ions will be also shifted to the inner of cells under action of the difference of electric- potential generated by the active transport in $\mathrm{Na}^{+}$ions. Therefore, the flowing of the $\mathrm{Na}^{+}$don't depend on the difference of electric -potential in the cell membrane (which is right in the case of below the interval of threshold values), but the flowing of $\mathrm{K}^{+}$ ions in inside cell is increased with the increase of the difference of electric-potential between both sides of cell membrane. This is just so-called the effect of the electric coupling between $\mathrm{Na}^{+}$and $\mathrm{K}^{+}$ion transport in the nerve systems. These results were confirmed in the researched results of the giant axons in the Grape snail obtained by r. Coase autumn, et al. [1-2,7-10].

From the above investigations we can conclude that we must use bio-energy to maintain the nonuniformal distribution of $\mathrm{Na}^{+}$ ions and $\mathrm{K}^{+}$ions and their gradients of concentration on the cell membrane using the sodium pump and potassium pump as well as the bio-energy released by hydrolysis reaction of ATP molecules. It is very interesting that these processes can go on and be finished simultaneously in these ionic pumps under action of the bio-energy. Therefore, we concluded that there are not works of the sodium pump and potassium pump as well as life activity of cell without the bio-energy.

\section{The form of action electric-potential under action of bio- energy in the nerve systems}

We know from the above investigations that the ions of $\mathrm{Na}^{+}, \mathrm{K}^{+}$ and $\mathrm{Cl}^{-}$are not the uniform distribution in the inside and outside cell membrane and their permeabilities across the membrane are also different, thus there is a resting electric- penitential of $-40--70 \mathrm{mV}$ in cell membrane, which arise from the motion of $\mathrm{K}^{+}$ ions. This means that the distribution of electric-potential of the membrane is the positive in outside membrane and negative in inside membrane, respectively, namely, the membrane is in the polar state in the resting state, which is just the state of polarization of cell membrane. However, when the cells are stimulated by a small electric signs, then its states and features will be changed. If a glass capillary electrode is inserted in the inside of the cells, then the depressions of the absolute value of negative differences of electric-potential between the inside and outside membrane e in a short period of time are inspected and observed. The values of decreases of the differences of electric-potential are further depressed with the increasing distance between measured and stimulated electrodes. This indicated that the electric-potential is localized. In general, when the stimuli are small, the changes of the negative potential in the inside membrane are small, which does not exceed a threshold. However, if the stimulated intensity is increased to exceed the threshold, then the electric-potentials of many excitable cells will be varied greatly and fast to become the positive values in inside membrane and negative in outside membrane from the above state at the time of $0.5 \mathrm{~ms}$. Subsequently, the change will increase continuously with increasing time, and makes the positive electric-potential reaches a maximum value (about 50 $\mathrm{mV}$ ) at a determined time, which is called the depolarization of the membrane. After this, the electric-potential will gradually decrease to original value at about $1 \mathrm{~ms}$, which is called stored polarization of the membrane. However, the deppessed state of the electric-potential cannot sopped and is deppessed continuously to a minimum, which is referred to as superpolarization. This changes of the electric-potential is referred to as an action electric-potential, which is represented in Figure 5.

The action electric-potential indicated clearly the changed features and rules of the electric- potential on cell membrane and ionic permeability across the cell membranes with varying time under action of external electric sign and influences of bio-energy. The variations can be described as follows. In the resting state the cell membrane is in the polarization state, its electric - potential is about $-40--60 \mathrm{mV}$, which is caused by the motions and the increases of permeability of $\mathrm{K}+$ ions. However, the depolarization is caused by the increases of permeability of $\mathrm{Na}^{+}$ions across the membrane under the influence of sodium pump and bio-energy released in the hydrolyses reaction of ATP molecules, which carry away $\mathrm{Na}^{+}$ions from the inside membrane into the outside membrane, thus the electric-potential in the outside membrane is increased, its strength is larger than that in inside membrane, thus the cell membrane is in the depolarization state, in which the electric- potential is lifted as shown in Figure 5. However, once the electric-potential reach a maximum, the potassium pump is started to work, it carries $\mathrm{K}^{+}$ions into the inside membrane from the outside membrane, thus the electric-potential is depressed and the cell membrane is in restored polarization state. the membrane is further in the superpolarization state with increasing time due to the inertial motion of $\mathrm{K}^{+}$ions, which are shown in Figure 5. Therefore, the electric-potential across the membrane of the neurons are determined and controlled by the relative permeabilities of ions of $\mathrm{Na}^{+}, \mathrm{K}^{+}$and $\mathrm{Cl}^{-}$, and their concentration gradient across membrane, but the bio-energy, 
which cause the concentration gradient of the ions in both sides of membranes, are carried out by the above ionic pumps, but their energies are obtained from the hydrolyses reaction of ATP molecules. The ionic pumps can not only pump initiatively out some ions but also bring these ions into the cells to maintain always the concentration gradient across membrane at a solid level. In this case the sodium pump can pump out three $\mathrm{Na}^{+}$in the outside of cell membrane to inside membrane, but the potassium pump can pump out two $\mathrm{K}^{+}$to inde membrane from outside membrane to change the electric potential on the cell membrane. When the variation of electric- potential of the axon membrane exceeds its threshold, then the action electric-potential is triggered, thus the excitement of the neurons occurs in the nervous system.

The action electric-potential is an electric signs, or speaking, it is essentially a nerve electric-impulse having certain electric-signs, its signs is great, rapid change and has a solid shape. It is exhibited and represented in the electric- potential having the constancies of size and form, its change of whole shape can be controlled in several milliseconds and depend not on the types and possess of the stimulation, and possess the nature of the "all or nothing". The action electrical- potential has not the distinction of size, but it can transport the nervous -biological information along the nerve fiber membranes in a stable wave form with certain frequency $i$, the higher of its frequency, the fast of its speed.

Once the action electric- potential reached the nerve terminal, then it will trigger and release some nerve neurotransmitters, in this case its secretion rate are also very higher. Obviously, this kind of action electric-potential is generated under synergistic action of $\mathrm{Na}^{+}$and $\mathrm{K}^{+}$ions in their channels. Because the open and close of two ion channels go on very accurately, then the contrary variation of the depolarization of following membrane and its difference of electrical -potential are quickly completed, if the action electricalpotential occurs, this means that the electrical-potential in inside cell is higher than that in outside cell. Subsequently, they back to the level of resting electrical- potential. In the change case of the action electrical-potential, it will be propagated along the axons from one neurons to other in the speed of $120 \mathrm{~m} / \mathrm{s}$ through the synapses up to the central nervous systems and the brain to cause nerve excitement.

Quite clearly, the action electrical-potential is determined by the motions and distributions of ions of $\mathrm{Na}+, \mathrm{K}+$ and $\mathrm{Cl}-$. Thus the electrical-potential across cell membrane under action of constant electric-field across membrane e is represented $l[1,8-10]$ by

$$
V=\phi=-\frac{K_{B} T}{F} \ln \left\{\frac{P_{K}\left[K^{+}\right]_{i}+P_{N a}\left[\mathrm{Na}^{+}\right]_{i}+P_{C l}\left[\mathrm{Cl}^{-}\right]_{0}}{P_{K}\left[\mathrm{~K}^{+}\right]_{0}+P_{N a}\left[\mathrm{Na}^{+}\right]_{0}+P_{C l}\left[\mathrm{Cl}^{-}\right]_{i}}\right\}
$$

where $\mathrm{P}_{\mathrm{K}} 、 \mathrm{P}_{\mathrm{Na}}$ and $\mathrm{P}_{\mathrm{Cl}}$ denote the permeabilities of $\mathrm{Na}, \mathrm{K}$ and $\mathrm{Cl}$ ions across the cell membrane, respectively, [ $]_{0}$ and []$_{\mathrm{i}}$ are the concentrations of ions in inside and outside membranes, respectively.
Evidently, the electrical-potential across cell membrane in Eq. (9) are related only to $\mathrm{Na}, \mathrm{K}$ and $\mathrm{Cl}$ ions, but only if the electric-potential in the inside membrane is lifted and the permeability of $\mathrm{Na}$ ions across the membrane from outside to inside is increased, then the values of the electric-potential across membrane will be changed to the positive from negative in the inner membrane. Subsequently, $\mathrm{Na}$ ions will infiltrate in the inner nerve fiber membrane, thus the electrical-potential will be lifted, which will lead to increases of osmotic quantity and speed of $\mathrm{Na}$ ions. However, when the electrical-potential in the inner membrane is lifted to certain value, a hysteresis effect occurs. In this case the permeability of $\mathrm{K}$ ions across the membrane is also increased. Because the concentration of Na ions in the inner fiber membrane are higher, then they can outwardly osmoses to the exterior of membrane. When the action electrical-potential is formed, the permeability of $\mathrm{K}$ ions will be increased and is faster than that of $\mathrm{Na}$ ions after $0.33 \mathrm{~ms}$, then the electrical-potential will return to original value $(-70 \mathrm{eV})$ from the maximum $(+50 \mathrm{mV})$. This effect cannot be varied although the influence of ion movement of penetration exist because it depend only the speed of motion of $\mathrm{K}$ ions to the outside of membrane. The $\mathrm{Na}$ ions in the nerve-fiber membrane, which are in the rising period of action electrical- potential in Figure 5 can pump out from the inner membrane by the sodium pump. This phenomenon can be verified in the following experiments.

a) Its maximum is increased, if the concentration of $\mathrm{Na}$ ions in the solution in outside membrane is lifted. On the contrary, if the concentration of $\mathrm{Na}^{+}$ions in outside membrane is depressed, then its maximum is decreased.

b) The maximum is decreased, when the concentration of $\mathrm{Na}$ ions in the solution in inside membrane is lifted.

c) The experiment found that the generation of action electrical-potential is accompanied always with the increases of shifted speed of $\mathrm{Na}$ ions along the direction of increase of the gradient of electrical -chemical potential. In the great nerve fibers in the squid, one time of the action electrical- potential will result the $\mathrm{Na}$ ions of $3.1 \mathrm{n} \mathrm{mol} . / \mathrm{cm}^{2}$ to come in the fiber cell.

The experimental results can explain qualitatively the increases of permeability of $\mathrm{Na}$ ions across the membrane, if the action electrical- potential appears. This can be verified by the experimental values of the permeability of the ions of $\mathrm{Na}^{+}, \mathrm{K}^{+}$and $\mathrm{Cl}^{-}$ across the membrane, for $\mathrm{Na}^{+}, \mathrm{K}^{+}$and $\mathrm{Cl}^{-}$their proportions are from 1:0.04:0.45 in resting case to 1:20:0.45 in the depolarization state and appearing of action electrical- potential. This experimental result affirm that the rising period of action electrical- potential or occurring of its depolarization effect in Figure 5 arises from the lifting of concentration or penetration of $\mathrm{Na}$ ions across membrane. Thus this verified that the analyses and results mentioned above are correct. 
In practice, the experiment of the radioactive tracer of sodium atomic motion in the squid indicated that the flowed quantities of movements of $\mathrm{Na}$ and $\mathrm{K}$ ions are small in its generated period, such as in the giant axon of the squid, about (3-4) $\times 10^{-12} \mathrm{~mol} /$ $\mathrm{cm}^{2}$ of $\mathrm{Na}^{+}$are shifted and absorbed only, the same number of $\mathrm{K}^{+}$ ions are lost and moved during the action electrical- potential or the period of electric-impulse. This indicated that the variation of concentration of $\mathrm{Na}$ or $\mathrm{K}$ ions are very small, are only one over one hundred thousand of the total numbers during one period of impulse of action electrical-potential. This is due to the inertia effect of depolarization functions of the membrane, which results in the depolarization effect of the membrane. In this case the nerve fiber membrane cannot occur second reaction of stimulus, only if the permeability of these ions are restored to original state, these ions can react to external stimulus after the time of 0.5-2 ms. Otherwise, the temperature can also greatly influence the above changes. If the temperature is varied from $6^{\circ} \mathrm{C}$ to $7{ }^{\circ} \mathrm{C}$, then the permeability of $\mathrm{K}$ ions increases, but the loss of $\mathrm{Na}$ ions is increased, thus the amplitude of the action electrical-potential will be decreased more. The above results manifest clearly that the bio-energy released in the hydrolysis reaction of ATP molecules plays key and important parts in works of sodium pump and potassium pump as well as the generation of action electrical-potential, there are not works of sodium pump and potassium pump as well as the generation of action electrical-potential without the bio-energy.

\section{The transport of the nerve impulsion}

We now study the concrete process of form of the action electrical-potential and its transfer along single nerve fiber membranes, which can be represented in Figure 6. In the front before part of the excited area or first end the cell membrane is in the restored polarization state, which is denoted in "static membrane "in this figure. Subsequently, the cell membrane is in a polarization state due to the stimulus of externally applied electrical-signs, such as electric current or field, in which the sodium current is formed due to the action of sodium pump and the bio-energy, meanwhile, where the electric-potential of the membrane is also varied to the negation in the outside membrane from the positive in the inside membrane. In the linkage region between the excited area and non-excited area there are the lifting of the electrical-potential of membrane and its depolarization due to that the sodium current flow over the membrane from inner to exterior in the non-excited area. When the electrical-potential of membrane reach the threshold value in this region, then the action electrical-potential occur in new excited area. In the subsequent part of the excited area the potassium current will also occurs due to form of the potassium pump and influence of the bio-energy, which makes the membrane restore to the polarize state. In this case the current, which is started from original excited area, makes the cell membrane reach the threshold in new region, thus one new excitation and corresponding new action electrical- potential are formed, its formed process can be denoted in the excitation depolarization - restored polarization, which is shown in Figure 6. This process is gone repeatedly on, then the transfer of action electrical- potential along the nerve membranes is appeared and carried out. In this case. This is just the mechanism of the transfer of action electrical- potential.

\section{References}

1. Pang Xiao-Feng (2007) Biophysics, Press of University of electronic science and Technology of China, Chengdu.

2. WL Liu (1994) Concise biophysics. Advanced education Press, Beijing.

3. JD Watson, FHC Crick (1953) Molecular Structure of Nucleic Acids: A Structure for Deoxyribose Nucleic Acid. Nature 171: 737-738.

4. Q Weaver (2000) Molecular biology, Boston, MC Graw Hill.

5. WX Zhu, L Li (2000) Modern molecular biology. Beijing, Advanced Education Press.

6. M Peyrard, S Cuesta-L [172] NS Cia (1992) Nerve biology, Science Press, Shanghai.

7. DA Baylor, AL Hodgkin, JD, Lamb (2003) J Physiol. 234: 163.

8. AL Hodgkin, AL Huxley, AF (1952) A quantitative description of membrane current and its application to conduction and excitation in nerve. J Physiol 117: 500-544.

9. AL Hodgkin, RD Keynes (1953) J Physiol 128: 28.

10. L Hodgkin, RD Keynes (1955) The potassium permeability of a giant nerve fibre. J Physiol 128(1): 61-88.
This work is licensed under Creative Commons Attribution 4.0 License

To Submit Your Article Click Here: Submit Article
DOI: 10.32474/OAJBEB.2018.02.000146.

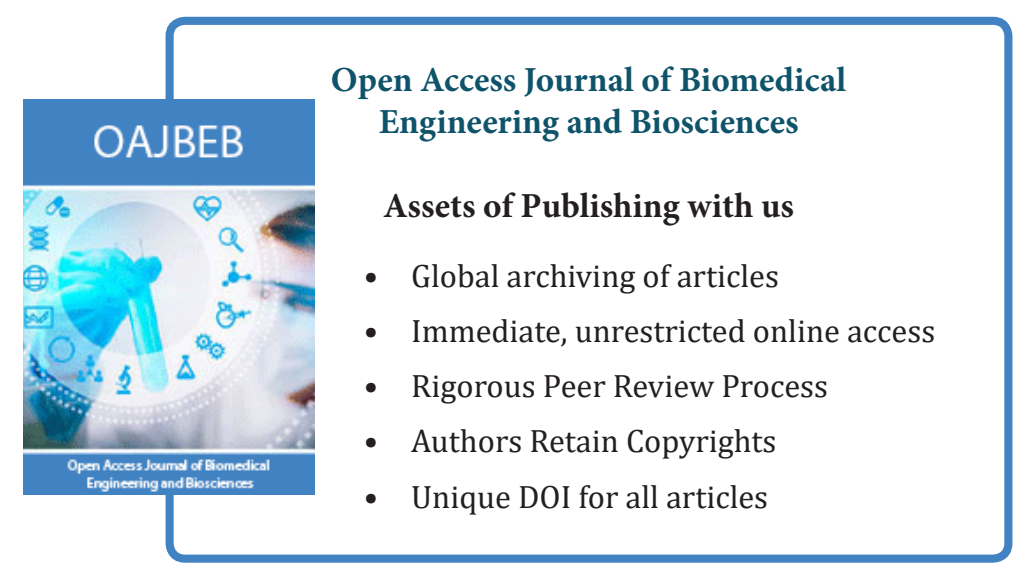

